\title{
Calidad de vida en la inclusión educativa de niños y ado- lescentes con discapacidad intelectual.
}

\author{
Quality of life in the educational inclusion of children and adolescents with \\ intellectual disability \\ Beleño- Melo, Betsy-Patricia *1.
}

Como citar este artículo: Beleño- Melo, Betsy-Patricia. Calidad de vida en la inclusión educativa de niños y adolescentes con discapacidad intelectual. Revistas Signos Fónicos.2019; 5(1):60-72.

Correspondencia autor: becho2483@gmail.com (Betsy Patricia Beleño Melo)

Recibido: Enero 28, 2019.

Aprobado: Mayo 6, 2019.

\begin{abstract}
RESUMEN
INTRODUCCIÓN: el objetivo es determinar la relación existe entre la calidad de vida de los niños/a y adolescentes con discapacidad intelectual de una institución educativa y un centro de rehabilitación de un barrio de la ciudad de Cúcuta, por medio de la escala KidsLife, y de esta forma dar respuesta a la pregunta de investigación. MÉTODOS: Corresponde a un estudio cuantitativo de tipo correlacional, de corte trasversal cuya muestra estuvo conformada por 24 sujetos de una institución educativa y un centro de rehabilitación. RESULTADOS: se encontraron diferencias significativas en la dimensión de bienestar fisco con un puntaje de 12 para no escolarizados y 8,5 para los escolarizados, a nivel general no se encontró diferencias significativas en el perfil de calidad, el de inclusión educativa obtuvo un puntaje de 9,667 y el otro colectivo de 9,25. ANÁLISIS Y DISCUSIÓN: la calidad de vida está dada por un modelo multidimensional que está relacionado tanto con factores personales como ambientales. CONCLUSIONES: Al evaluar la calidad de vida se debe tener en cuenta los diferentes contextos en los que se desenvuelve el individuo.
\end{abstract}

PALABRAS CLAVE: Calidad de vida, discapacidad intelectual, niño, adolescente.

\begin{abstract}
INTRODUCTION: The objective is to determine the relationship between the quality of life of children and adolescents with intellectual disabilities in an educational institution and a rehabilitation center in a neighborhood of the city of Cúcuta, through the KidsLife scale, and in this way answer the research question. METHODS: Corresponds to a quantitative study of correlational type, cross-sectional whose sample consisted of 24 subjects of an educational institution and a rehabilitation center. RESULTS: Significant differences were found in the fiscal welfare dimension with a score of 12 for out-of-school children and 8.5 for school-age children. At the general level, no significant differences were found in the quality profile, educational inclusion scored a score of 9.667 and the other collective of 9.25. ANALYSIS AND DISCUSSION: The quality of life is given by a multidimensional model that is related to both personal and environmental factors. CONCLUSIONS: When evaluating the quality of life, the different contexts in which the individual develops should be taken into account.
\end{abstract}

KEYWORDS: Quality of Life, Intellectual Disability, Child, Adolescent.

1 *Fonoaudióloga, becho2483@gmail.com, Orcid: 0000-0002-0183-2509, Hospital Hernando Quintero Blanco E.S.E., Colombia. 


\section{INTRODUCCIÓN}

La discapacidad es un concepto que viene a estar determinada por la interacción que resulta del vínculo de una persona con el contexto en que se desenvuelve, a su vez, está relacionada con las diversas condiciones de salud de cada individuo, con los diferentes factores personales y externos que se encuentran inmersos dentro del entorno que lo rodea, en donde su funcionalidad está directamente enlazada a las circunstancias en las que la persona vive(1-6). Este término maneja una clasificación, entre las que se encuentra el grupo de discapacidades mentales, aquí está inmerso el retardo mental o como actualmente se le conoce "discapacidad intelectual"(7)(8)(9)(10), esta se caracteriza por presentar un nivel de funcionamiento intelectual por debajo de la media, en relación a los individuos de su misma edad; así mismo, presentan dificultad en las habilidades y conductas adaptativas, conceptuales, sociales y prácticas(1)(11). En esta medida, comprender el concepto de discapacidad intelectual y todo lo que la involucra es importante, ya que esto, va a permitir conocer y entender de qué forma esta condición puede llegar a influenciar en el nivel de calidad de vida de una persona.

En relación con esto último, se tiene el concepto de calidad de vida, definida por la Organización Mundial de la Salud (OMS) en 1994, como esa forma en el que la persona se percibe dentro de un contexto cultural, incluidos los sistemas de valores en el cual vive, relacionando todo eso con sus objetivos, expectativas, estándares e intereses de vida(12)así como los criterios básicos que se requieren para la adaptación, construcción y aplicación de instrumentos, para su evaluación y medición. Para ello se recopiló información reportada en diferentes bases de datos y posteriormente se examinó por medio de la elaboración de resúmenes analíticos especializados (RAES (13)(14)(15)(11)(15)(16)(17).

De acuerdo con los razonamientos que se han venido realizando, Schalock y Verdugo, definen la calidad de vida desde un concepto multidimensional, que está compuesto por unas dimensiones, y estas a su vez van a variar de una persona a otra, de grupo a grupo y de lugar a lugar, además, posee componentes objetivos y subjetivos, y está influenciado por factores tanto ambientales, como personales y de interacción. Éste término se basa en el grado tanto de experiencias positivas como negativas que tienen los sujetos, reflejándose dentro de los contextos culturales y sociales que rodean a los individuos, los cuales son necesarios para su bienestar personal(12), así como los criterios básicos que se requieren para la adaptación, construcción y aplicación de instrumentos, para su evaluación y medición. Para ello se recopiló información reportada en diferentes bases de datos y posteriormente se examinó por medio de la elaboración de resúmenes analíticos especializados RAES (18)(19)(20)(21) (22)(23).

El término de calidad de vida ha ido adquiriendo una importancia e interés especial dentro de la investigación y en la práctica de los servicios sociales en general, educativos y sanitarios. Además, conocer la calidad de vida de un individuo resulta interesante, ya que a menudo se critica, se admira o se sobreestima a las personas sin conocer su vida, el contexto en que se desenvuelve, y un sin número de cosas que pueden estar influyendo entorno en esta.(11)(24)(25). Durante las tres últimas décadas, los esfuerzos llevados a cabo durante este tiempo han permitido que el concepto de calidad de vida haya evolucionado desde una noción sensibilizadora hasta convertirse en un agente de cambio social y organizacional(26).

Teniendo en cuenta algunos estudios hechos en este tema, se tiene que en Chile, son poco los estudios publicados en calidad de vida de infantes y adolescentes, y sobre todo en aquellos que describen valores que puedan servir de referencia para el uso clínico o epidemiológico, facilitando así la utilización de la evaluación de indicadores como el bienestar, la salud percibida entre otros(27).

Revista Científica Signos Fónicos, 2019,5(1): 60-72. ISNN 2422-1716. 
Así mismo, en Colombia los estudios relacionados con la evaluación de la calidad de vida han tenido un incipiente desarrollo. Las primeras aproximaciones al tema se hicieron a partir de las encuestas sobre calidad de vida que solamente miden condiciones de vida, consideradas apenas una dimensión de lo que hoy se entiende por calidad de vida. El primer estudio fue desarrollado para la ciudad de Bogotá en 1991 y posteriormente en los años 1993, 1997, 2003 y 2008 con cobertura nacional(28)(23).

Inicialmente, las primeras investigaciones que se realizaron sobre calidad de vida se empezaron en población de personas adultas, y poco a poco se ha venido desarrollando estudios relacionados con la calidad de vida de niños y adolescentes principalmente en edad escolar, pero la mayor parte de la información que se evidencia está relacionada con población adulta. Es de este modo, como el concepto de calidad de vida en la infancia empezó a aplicarse en contextos educativos (Schalock y Verdugo, 2003), siendo el Informe Warnock (Warnock, 1978) el inicio del movimiento inclusivo en la educación, el cual proponía que los objetivos de la educación debían ser iguales para todos los alumnos y que se debía proporcionar apoyo para que todas las personas, independientemente de sus características, alcanzarán metas personales(13)(29)(30).

En Colombia existe la ley estatutaria 1618 de 2013, en la cual se garantizan los derechos de las personas con discapacidad y de su inclusión a los centros educativos y a los diferentes entes de carácter público, esto con el objeto que promover la participación, igualdad, atención en los servicios de salud, protección, empleo(31).

Por lo anterior, la investigación tiene como objetivo determinar la relación entre un aumento de la calidad de vida de los niños/a y adolescentes con discapacidad intelectual de una institución educativa y un centro de rehabilitación de un barrio de la ciudad de Cúcuta, por medio de la escala KidsLife, que es un instrumento desarrollado por M. Verdugo, B. Martínez, L.E. Sánchez y R.L. Schalock, basado en el modelo multidimensional de Sharlock y Verdugo (2002/2003) que permite la evaluación objetiva y subjetiva de la calidad de vida en las personas. A partir de esto surge el siguiente interrogante ¿existe relación entre la calidad de vida de los niños/a y adolescentes con discapacidad intelectual de una institución educativa y un centro de rehabilitación de un barrio de la ciudad de Cúcuta?

\section{MÉTODOS}

En la investigación desarrollada se establece un enfoque cuantitativo, de tipo correlacional, de corte transversal; llevada a cabo en un colegio público en el barrio Antonia Santos y en dos centros de rehabilitación de la ciudad de Cúcuta.

Los estudios cuantitativos se caracterizan por ser un proceso secuencial y probatorio, donde la recolección de datos es utilizada para probar hipótesis basándose en la medición numérica y el análisis estadístico, esto con el fin de establecer pautas de comportamiento y probar teorías(17).

Ahora bien, los diseños de investigación transversal son aquellos en los que la recolección de los datos se da en un solo momento, en un tiempo único; y tiene como finalidad describir variables y analizar su incidencia e interrelación en un momento dado(17)(18).

\section{Instrumento}

Para llevar a cabo el estudio de la evaluación de la calidad de vida de los niños y adolescentes con discapacidad intelectual, se utilizó la escala KidsLife, teniendo en cuenta que es un instrumento que 
permite la evaluación multidimensional de la calidad de vida, basada en el modelo de ocho dimensiones de Schalock y Verdugo $(2002,2012)(19)$.

La escala está diseñada para ser aplicada a personas desde los 4 hasta los 21 años de edad con discapacidad intelectual, permite obtener información importante acerca de los diferentes contextos en los que a diario se desenvuelve el individuo. Consta de datos de la persona evaluada, datos del informador principal y de 96 ítems cada uno con cuatro opciones de respuesta (nunca, a veces, frecuentemente y siempre) distribuidos entre las dimensiones a evaluar:

La inclusión social hacer referencia a ir a lugares de la ciudad o del barrio donde van otras personas y participar en sus actividades como uno más. Sentirse miembro de la sociedad, sentirse integrado, contar con el apoyo de otras personas; evalúa indicadores de integración, participación, accesibilidad y apoyos.

La autodeterminación, se relaciona con la toma de decisiones por sí mismo y tener oportunidad de elegir las cosas que quiere, cómo quiere que sea su vida, su trabajo, su tiempo libre, el lugar donde vive, las personas con las que está; tiene en cuenta indicadores como autonomía, metas, opiniones y preferencias personales.

El bienestar emocional, se refiere al hecho de sentirse tranquilo, seguro, sin agobios, no estar nervioso; esta dimensión mide indicadores de satisfacción con la vida, autoconcepto, afectividad y emociones, estabilidad y salud mental.

El bienestar físico, tiene que ver con tener buena salud, sentirse en buena forma física, tener hábitos de alimentación saludables; mide indicadores de descanso, sueño, higiene, alimentación, atención sanitaria y salud física.

El bienestar material, se refiere a tener suficiente dinero para comprar lo que se necesita y se desea tener, tener una vivienda y lugar de trabajo adecuados; evalúa indicadores de vivienda, nuevas tecnologías y ayudas técnicas, bienes materiales y servicios.

Derechos, se refiere a ser considerado igual que el resto de la gente, que le traten igual, que respeten su forma de ser, opiniones, deseos, intimidad, derechos; mira indicadores como el ejercicio de derechos, intimidad, confidencialidad, respeto y conocimientos el sobre estos.

Desarrollo personal, se relaciona con la posibilidad de aprender distintas cosas, tener conocimientos y realizarse personalmente; tiene en cuenta indicadores de resolución de problemas, actividades de la vida diaria y enseñanza/aprendizaje; y como ultima dimensión se tiene la de relaciones interpersonales, esta se refiere a relacionarse con distintas personas, tener amigos y llevarse bien con la gente (vecinos, compañeros, etc.); mide indicadores de comunicación, relaciones familiares, amigos, Compañeros y Sociedad.

Este instrumento se encuentra validado por la Universidad de Oviedo e Instituto Universitario de Integración en la Comunidad (INICO). Tiene como finalidad identificar el perfil de calidad de vida de la persona, y cuenta con evidencias necesarias para su validez y fiabilidad, que a su vez servirán para llevar a cabo prácticas basadas en la evidencia y la elaboración de planes individuales de apoyo (19)(18)(5).

Para el estudio, se tuvo en cuenta los siguientes criterios de inclusión y exclusión: 
TABLA 1. Criterios de inclusión y exclusión.

\begin{tabular}{ll}
\multicolumn{1}{c}{ INCLUSIÓN } & \multicolumn{1}{c}{ EXCLUSIÓN } \\
\hline Edades entre los 4 y 21 años & Menores de 4 y mayores a 21 años \\
\hline $\begin{array}{l}\text { Tener diagnóstico de discapacidad intelec- } \\
\text { tual }\end{array}$ & No tener diagnóstico de discapacidad intelectual \\
\hline $\begin{array}{l}\text { Que el informante tuviera por lo menos seis } \\
\text { meses de estar conociendo a la persona }\end{array}$ & $\begin{array}{l}\text { Que el informante tuviera menos de seis meses } \\
\text { de estar conociendo a la persona }\end{array}$ \\
\hline
\end{tabular}

Fuente: Los Autores

La población objeto de estudio estuvo conformada por 24 participantes, de los cuales unos pertenecen a la institución educativa, y los otros no son escolarizados, ambos de la ciudad de Cúcuta.

Para llevar a cabo el proceso de la recolección de datos en su totalidad, se hizo necesario establecer tres fases, en la primera fase se desarrolló el proceso de revisión documental, esto con el fin investigar acerca de la calidad de vida de las personas con discapacidad intelectual y sobre la utilidad de la escala KidsLife. Para la selección de la información se tuvo en cuenta que los artículos tuvieran relación con el tema de trabajo expuesto, con una ventana de tiempo de 10 años, y que estuvieran indexados en bases de datos confiables y precisos.

En la segunda fase se realizó la aplicación de la escala KidsLife, en primer lugar, se dio a conocer el consentimiento informado, donde se les explicó en qué consistía el proceso, aclarándoles que en el momento que quisieran se podían retirar de la investigación. Seguido a esto se procede a dar inicio a la aplicación del instrumento a padres o cuidadores, los cuales llevaban más de seis meses de estar conociendo y conviviendo con la persona objeto de estudio; este instrumento tiene ocho dimensiones que dan cuenta del perfil de calidad de vida de cada individuo. El proceso se llevó a cabo de la siguiente manera:

- Primero se diligencio la información personal de la persona evaluada.

- Segundo se diligencio la información de del informador principal.

- Tercero se diligenciaron los 96 ítems correspondientes a las dimensiones a evaluar.

\section{Tercera fase: proceso de análisis}

Esta fase se inicia con la revisión y organización de los datos, aquí se realiza el conteo de cada una de las respuestas dadas en cada dimensión, para asignar la puntuación a cada uno de los individuos de acuerdo a los baremos establecidos por la escala, con el objetivo de obtener la puntuación total directa, puntuación estándar, percentiles, índices de calidad de vida general y percentil de esta misma por cada una de las dimensiones. Lo anterior, fue fundamental para conseguir las medidas de tendencia central ya que de estas últimas se basaron los resultados de la investigación.

Para el procesamiento y análisis de la información recolectada se usó el software estadístico SPSS versión 22.0 en español, el cual permitió realizar procesos estadísticos, para ayudar a crear y trabajar fundamentalmente en la abstracción y estudio de los resultados, además se hizo un análisis descriptivo de la información para caracterizar la población. Se empleó la prueba de Mann Whitney para comparar las medidas de calidad de vida de los grupos bajo estudio, la cual es una prueba no paramétrica que se aplica en el caso de dos muestras independientes(32). Teniendo en cuenta el estudio se evidencia un nivel de confiabilidad del $95 \%$ y de significancia del $5 \%$. 
El presente estudio realizado en el área de la salud cumple con los parámetros propuestos en la resolución 8430 de 1993, en lo concerniente a las normas científicas, técnicas y administrativas; cuando dice que se debe proteger la información de las personas de estudio con carácter reservado y con fines académicos, esta investigación no representa ningún riesgo debido a que emplea técnicas y métodos de investigación documental retrospectiva en la que se utilizó una escala para medir la calidad de vida, sin realizar ningún tipo de intervención(33).

\section{RESULTADOS}

En los resultados obtenidos se presentan los principales hallazgos del estudio, distribuidos de la siguiente manera: clasificación de los sujetos bajo estudio de acuerdo a la edad, sexo y tipo de escolarización, clasificación de los sujetos bajo estudio de acuerdo a el sexo, nivel de discapacidad intelectual y tipo de escolarización, clasificación de los sujetos bajo estudio de acuerdo a el sexo, nivel de necesidades de apoyo y tipo de escolarización, promedios estándar globales y perfil de calidad de vida, representación de la dimensión bienestar físico de acuerdo al tipo de escolarización y por último resultados de la prueba Mann Whitney.

\begin{tabular}{clllllll} 
& \multicolumn{4}{c}{ Tipo de escolarización } \\
& \multicolumn{3}{c}{ Escolarizados } & \multicolumn{3}{c}{ No escolarizados } \\
\cline { 2 - 7 } & \multicolumn{3}{c}{ EDAD_CATEG } & \multicolumn{3}{c}{ EDAD_CATEG } \\
\cline { 2 - 7 } & $\mathbf{6}-\mathbf{1 5}$ & $>=16$ & $<=5$ & $\mathbf{6}-\mathbf{1 5}$ & $>=16$ \\
\hline SEXO & $\begin{array}{l}\text { Frecuen- } \\
\text { cia }\end{array}$ & $\begin{array}{l}\text { Frecuen- } \\
\text { cia }\end{array}$ & $\begin{array}{l}\text { Frecuen- } \\
\text { cia }\end{array}$ & $\begin{array}{l}\text { Frecuen- } \\
\text { cia }\end{array}$ & $\begin{array}{l}\text { Frecuen- } \\
\text { cia }\end{array}$ & $\begin{array}{l}\text { Frecuen- } \\
\text { cia }\end{array}$ \\
\hline $\begin{array}{c}\text { Feme- } \\
\text { nino }\end{array}$ & 0 & 7 & 1 & 1 & 2 & 1 \\
\hline $\begin{array}{c}\text { Mas- } \\
\text { culino }\end{array}$ & 0 & 4 & 0 & 1 & 5 & 2 \\
\hline
\end{tabular}

TABLA 2. clasificación de los sujetos bajo estudio de acuerdo a: la edad, sexo y tipo de escolarización.

1. En la se observan los datos correspondientes al tipo de escolarización, edad y sexo, donde se muestra que, en el grupo de escolarizados, la edad que predomina es la de 6 a 15 años compuesta por 7 sujetos de sexo femenino y 4 del masculino, seguido de los $>=16$ con 1 para el femenino, para un total de 12 sujetos; mientras que, en la población de no escolarizados, sobresalen las edades de 6 a 15 años, representado por 5 preadolescentes de sexo masculino y 2 del femenino, luego sigue la edad >= 16 con 2 del masculino y 1 del femenino, por último se tienen los $<=5$ años con 1 de masculino y 1 de femenino para un total de 12 individuos. 
TABLA 3. clasificación de los sujetos bajo estudio de acuerdo a: el sexo, nivel de discapacidad intelectual y tipo de escolarización.

\begin{tabular}{lcccccccc}
\hline \multicolumn{10}{c}{ Tipo de escolarización } \\
\hline \multicolumn{7}{c}{ Escolarizados } & \multicolumn{5}{c}{ No escolarizados } \\
\hline & Leve & $\begin{array}{c}\text { Mode- } \\
\text { rado }\end{array}$ & Severo & Total & Leve & $\begin{array}{c}\text { Mode- } \\
\text { rado }\end{array}$ & $\begin{array}{c}\text { Seve- } \\
\text { ro }\end{array}$ & Total \\
\hline & $\begin{array}{c}\text { Fre- } \\
\text { cuen- } \\
\text { cia }\end{array}$ & $\begin{array}{c}\text { Fre- } \\
\text { cuencia }\end{array}$ & $\begin{array}{c}\text { Fre- } \\
\text { cuencia }\end{array}$ & $\begin{array}{c}\text { Fre- } \\
\text { cuencia }\end{array}$ & $\begin{array}{c}\text { Fre- } \\
\text { cuen- } \\
\text { cia }\end{array}$ & $\begin{array}{c}\text { Frecuen- } \\
\text { cia }\end{array}$ & $\begin{array}{c}\text { Fre- } \\
\text { cuen- } \\
\text { cia }\end{array}$ & $\begin{array}{c}\text { Fre- } \\
\text { cuencia }\end{array}$ \\
\hline Sexo & 7 & 1 & 0 & 8 & 3 & 0 & 1 & 4 \\
\hline $\begin{array}{l}\text { Feme- } \\
\text { nino }\end{array}$ & 7 & 1 & 0 & 4 & 4 & 3 & 1 & 8 \\
\hline $\begin{array}{l}\text { Mascu- } \\
\text { lino }\end{array}$ & 3 & & & & & & & \\
\hline
\end{tabular}

Fuente: Los autores

En la tabla se muestran los datos correspondientes al tipo de escolarización, el nivel de discapacidad y el sexo, indicando que en los sujetos del grupo que pertenecen a la inclusión educativa, se evidencia que el nivel de discapacidad que prevalece es el leve con 7 individuos de sexo femenino y 3 del masculino, seguido del moderado con 1 del femenino y 1 del masculino, para un total de 12 personas; con respecto al grupo de no escolarizados, en este predomina el nivel de discapacidad leve con 4 personas de sexo masculino y 3 del femenino, continuado del moderado con 3 de sexo masculino y por último el severo con 1 femenino y 1 masculino, para un total de 12 sujetos.

TABLA 4. Clasificación de los sujetos bajo estudio de acuerdo a: el sexo, nivel de necesidades de apoyo y tipo de escolarización.

\begin{tabular}{|c|c|c|c|c|c|c|c|c|c|}
\hline & \multicolumn{9}{|c|}{ Tipo de escolarización } \\
\hline & \multicolumn{5}{|c|}{ Escolarizados } & \multicolumn{4}{|c|}{ No escolarizados } \\
\hline & \multicolumn{5}{|c|}{ Nivel de necesidades de apoyo } & \multicolumn{4}{|c|}{ Nivel de necesidades de apoyo } \\
\hline & & $\begin{array}{c}\text { Exten- } \\
\text { so }\end{array}$ & $\begin{array}{c}\text { Gene- } \\
\text { raliza- } \\
\text { do }\end{array}$ & $\begin{array}{l}\text { Inter- } \\
\text { miten- } \\
\text { te }\end{array}$ & $\begin{array}{l}\text { limita- } \\
\text { do }\end{array}$ & $\begin{array}{c}\text { Exten- } \\
\text { so }\end{array}$ & $\begin{array}{c}\text { Gene- } \\
\text { raliza- } \\
\text { do }\end{array}$ & $\begin{array}{l}\text { inter- } \\
\text { miten- } \\
\text { te }\end{array}$ & $\begin{array}{l}\text { limi- } \\
\text { tado }\end{array}$ \\
\hline & & $\begin{array}{l}F r \text { e - } \\
\text { cuencia }\end{array}$ & $\begin{array}{l}\mathrm{Fre}- \\
\text { cuencia }\end{array}$ & $\begin{array}{l}\mathrm{F} r \mathrm{e}- \\
\text { cuencia }\end{array}$ & $\begin{array}{l}\mathrm{F} \mathrm{re} \mathrm{-} \\
\text { cuencia }\end{array}$ & $\begin{array}{l}\mathrm{F} \mathrm{r} \mathrm{e} \mathrm{-} \\
\text { cuencia }\end{array}$ & $\begin{array}{l}\text { F r e - } \\
\text { cuencia }\end{array}$ & $\begin{array}{l}\mathrm{Fr} \text { e - } \\
\text { cuencia }\end{array}$ & $\begin{array}{l}\mathrm{F} r \mathrm{e} \\
\text { cuencia }\end{array}$ \\
\hline \multirow[t]{2}{*}{ Sexo } & $\begin{array}{l}\text { Feme- } \\
\text { nino }\end{array}$ & 0 & 0 & 2 & 6 & 0 & 1 & 0 & 3 \\
\hline & $\begin{array}{l}\text { Mas - } \\
\text { culino }\end{array}$ & 0 & 0 & 1 & 3 & 1 & 1 & 2 & 4 \\
\hline
\end{tabular}

Fuente: Los autores

En la tabla se perciben los datos correspondientes al tipo de escolarización, nivel de necesidades de apoyo y sexo, donde se indica que en el grupo de individuos que se encuentra dentro del sistema educativo prevalece un nivel de necesidad de apoyo limitado, compuesto por 6 estudiantes de sexo femenino y 3 de sexo masculino, seguido del intermitente con 2 de sexo femenino y 1 de masculino, para un total de 12 personas; en relación a la población de no escolarizados el nivel de necesidades de 
apoyo que sobresale, es el limitado compuesto por 4 personas de sexo masculino y 3 de sexo femenino, continuado del intermitente con 2 de sexo masculino, seguido del generalizado con 1 femenino y 1 masculino y por último el extenso con 1 del sexo masculino, para un total de 12 sujetos.

TABLA 5. Relación de promedios estándar globales y perfil de calidad de vida de por grupo.

\begin{tabular}{|c|c|c|c|c|c|c|}
\hline & Escolarizad & & & No escolariza & dos & \\
\hline $\begin{array}{l}\text { Dimensio- } \\
\text { nes }\end{array}$ & $\begin{array}{l}\text { Promedio } \\
\text { estándar }\end{array}$ & $\begin{array}{l}\text { Valor máxi- } \\
\text { mo }\end{array}$ & $\begin{array}{l}\text { Valor míni- } \\
\text { mo }\end{array}$ & $\begin{array}{l}\text { Promedio } \\
\text { estándar }\end{array}$ & $\begin{array}{l}\text { Valor máxi- } \\
\text { mo }\end{array}$ & $\begin{array}{l}\text { Valor mí- } \\
\text { nimo }\end{array}$ \\
\hline $\begin{array}{l}\text { Inclusión } \\
\text { social }\end{array}$ & 12 & 14 & 6 & 9 & 17 & 5 \\
\hline $\begin{array}{l}\text { Autodeter- } \\
\text { minación }\end{array}$ & 9,5 & 11 & 7 & 8 & 14 & 2 \\
\hline $\begin{array}{l}\text { Bienestar } \\
\text { emocional }\end{array}$ & 10 & 13 & 4 & 10 & 14 & 5 \\
\hline $\begin{array}{l}\text { Bienestar } \\
\text { físico }\end{array}$ & 8,5 & 13 & 3 & 12 & 14 & 7 \\
\hline $\begin{array}{l}\text { Bienestar } \\
\text { material }\end{array}$ & 11 & 14 & 7 & 9,5 & 12 & 1 \\
\hline Derechos & 8 & 15 & 1 & 8,5 & 14 & 4 \\
\hline $\begin{array}{l}\text { Desarrollo } \\
\text { personal }\end{array}$ & 11,5 & 14 & 9 & 11,5 & 14 & 3 \\
\hline $\begin{array}{l}\text { Relaciones } \\
\text { interperso- } \\
\text { nales }\end{array}$ & 10 & 13 & 5 & 10 & 13 & 7 \\
\hline $\begin{array}{ll}\text { Perfil } & \text { de } \\
\text { calidad } & \text { de } \\
\text { vida } & \end{array}$ & 9,667 & 11,057 & 8,276 & 9,25 & 11,178 & 7,313 \\
\hline
\end{tabular}

Fuente: Los Autores

En la tabla se observan los datos arrojados de forma global, correspondientes al tipo de escolarización relacionado con el valor promedio estándar, máximo y mínimo de cada dimensión, detallándose que, en los participantes pertenecientes al grupo de inclusión educativa, la dimensión con mayor puntuación fue la de inclusión social con un puntaje de 12, y la más baja fue la de derechos con un valor de 8 , así mismo, el indicador promedio en general del perfil de calidad de vida de este colectivo fue de 9,667; mientras que el grupo de no escolarizados, obtuvo la puntación más alta en la dimensión de bienestar físico, con un puntaje estándar de 12, y la más baja con 8 para autodeterminación, con un indicador promedio en general del perfil de calidad de vida de 9,25. 


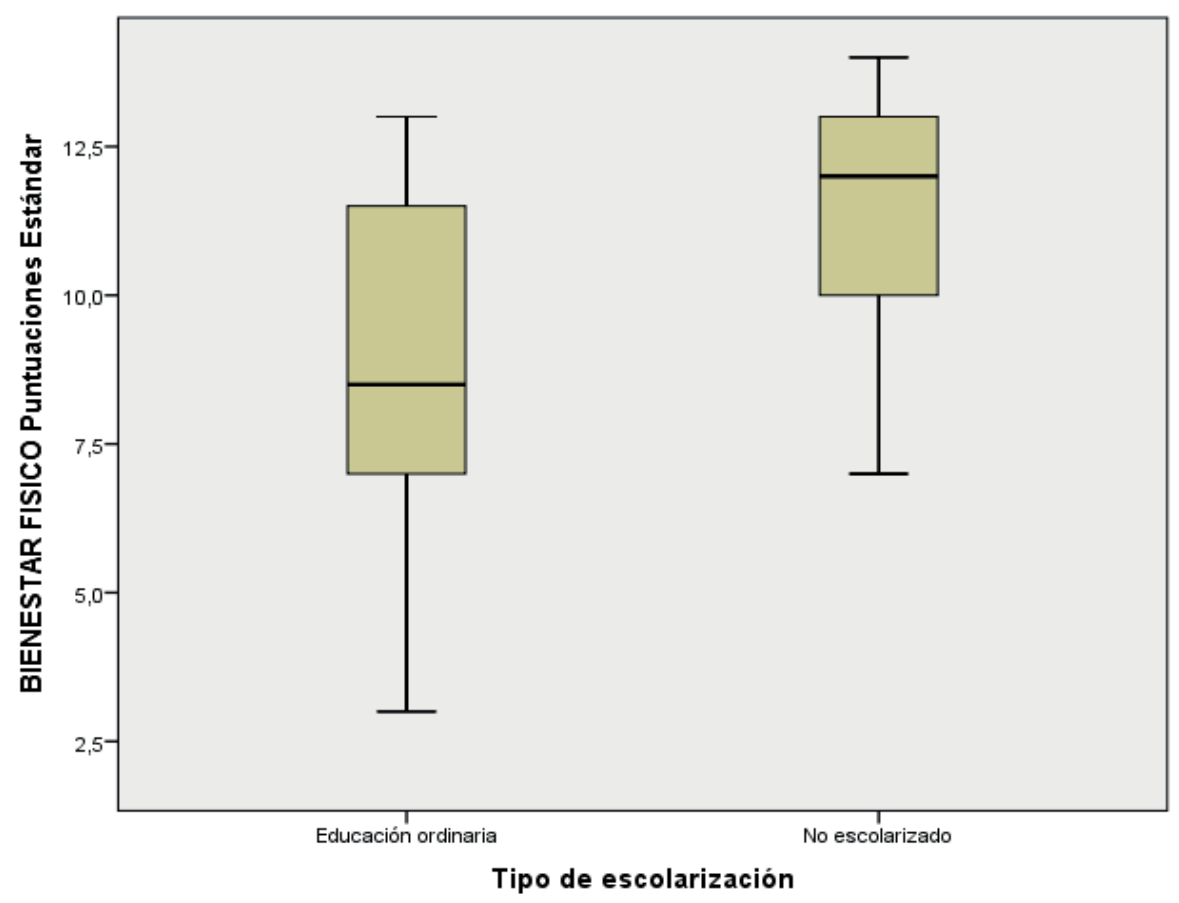

Imagen 1. Representación de la dimensión bienestar físico de acuerdo al tipo de escolarización.

En el grafico anterior se evidencia la representación de los datos correspondientes a la dimensión de bienestar físico, separada de acuerdo a la asistencia o no de los grupos a una institución educativa, donde se observa que aproximadamente el $50 \%$ de los individuos no escolarizados tienen un indicador promedio de calidad de vida de 12, en relación a los sujetos escolarizados que se encuentran con un indicador promedio de calidad de vida de 8,5. Todo lo anterior indica que en el grupo de no escolarizados existe poca variabilidad, es decir, los individuos en este grupo son muy parecidos entre sí, mientras que los sujetos del grupo de educación formal presentan una gran variabilidad, lo que quiere decir que existen más diferencias entre estos, de acuerdo al indicador de calidad de vida de dicha dimensión.

TABLA 6. Resultados Prueba Mann Whitney

\begin{tabular}{llll} 
& \multicolumn{3}{c}{ DIMENSIONES } \\
& P-Valor & Significa & Decisión \\
\hline TES.... & 0,02 & 0,05 & Rechazo H0 \\
\hline
\end{tabular}

Fuente: Los autores

En la tabla 6. Se observan los resultados de la prueba de Mann Whitney, es una prueba no paramétrica, la cual indicó que estadísticamente existen diferencias poco significativas en cuanto al indicador promedio del nivel de calidad de vida en ambos grupos de estudio, lo cual podía interpretarse que las diferencias entre las medias asociadas al indicador de calidad de vida son estadísticamente diferentes.

\section{ANÁLISIS Y DISCUSIÓN}

Este estudio evalúa la calidad de vida de los niños y adolescentes con discapacidad intelectual de escolarizados y no escolarizados, es así, como se parte de este concepto, tomando como referente el mo- 
delo multidimensional compuesto por ocho dimensiones, que apoyado en la teoría está relacionado con la conducta y la integración de los individuos al ambiente, abordando no solo el estado de salud sino todas las aristas donde se desenvuelve el ser humano incluido el nivel socioeconómico, bienestar personal y desarrollo social entre otros (34)(35).

Según los hallazgos estudiados y analizados en la presente investigación, en la dimensión de bienestar físico fue donde se obtuvo una diferencia significativa en relación a los dos grupos de estudio, evidenciándose mejor calidad de vida en los participantes del colectivo de no escolarizados; de acuerdo a esto, se percibe que los familiares de los sujetos evaluados ven más factible llevar a los niños a terapias para que puedan rehabilitarse, y de esta forma obtener buenas condiciones de salud. En referencia a lo anterior la teoría concuerda con los resultados obtenidos al afirmar que últimamente se han visto cambios relevantes en cuanto a la atención y cuidados de las personas con discapacidad, donde los servicios médicos preventivos y rehabilitadores son más integrales y tienen en cuenta todos los problemas de salud que pueden afectar al individuo, de esta manera se estaría logrando mayor bienestar físico (Gaite et al., 2008)(36)(37).

Por el contrario, el análisis de las dimensiones en cada uno de los grupos evaluados, arrojó que la población de escolarizados y no escolarizados presentan un indicador promedio, donde el grupo de inclusión educativa obtuvo un perfil de calidad de vida de 9,67 y el de no escolarizados de 9,25, con lo anterior se percibe que el asistir al colegio, así como a centros de rehabilitación, mejora la calidad de vida. En este sentido, la teoría concuerda con lo planteado por Ruden \& Gosch (2006), cuando afirma que en la medida en que la persona tenga la posibilidad de acceder a servicios que puedan elevar su bienestar, como es educación y salud, la calidad de vida mejorará(38).

En lo referente al nivel de necesidades de apoyo, en este estudio se observa que todas de las personas requieren de apoyos en su mayoría de forma limitada, es decir, estos individuos tienden a ser un poco más independientes a la hora de desarrollar actividades de la vida diaria, permitiendo de esta manera tener un funcionamiento más individual. De acuerdo a la teoría esta nos dice que los apoyos promueven el desarrollo, la educación, intereses y bienestar personal de una persona y mejoran el funcionamiento individual de la misma. (Luckasson, R., Borthwick-Duffy, S., Buntix, W.H.E., Coulter, D.L., Craig, E.M., Reeve, A., y cols., 2002)(39).

Al asociar la variable nivel de discapacidad con el género y el tipo de escolarización, se evidencia que el mayor número de personas pertenecen al sexo femenino, con un nivel de discapacidad intelectual leve, esto le va permitir a estos individuos que su desenvolvimiento e interacción en los diferentes contextos sea más favorable. Estos resultados coinciden con los obtenidos por CERMI (2010) y Unicef $(2013 a, b)$ en donde se plantea que, a menor nivel de discapacidad intelectual, menor será la dificultad para que estas personas puedan participar en actividades sociales y recreativas, así mismo, así mismo, habrá menor sobreprotección y podrán desarrollar una mayor autonomía personal(36).

En la dimensión de derechos se observó que ambos grupos se encuentran por debajo del promedio estándar, esto quiere decir que en esta población son vulnerados los derechos de estas personas, como, por ejemplo, desconocer los decretos, leyes y normas que los acobijan. Entre las que se encuentra la ley 1618 del 2013 por medio de la cual se establece las disposiciones para garantizar el pleno ejercicio de los derechos de las personas con discapacidad(40) (41) (42) (43).

\section{CONCLUSIONES}

En la presente investigación se concluye que no existe una diferencia significativa ente el grupo de escolarizados y no escolarizados, observando que asistir tanto a una institución educativa como a centros de rehabilitación permite mejorar la calidad de vida de las personas en situación de discapacidad.

De acuerdo a la dimensión de bienestar físico se observó que existe una diferencia significativa en ambos grupos, determinado porque los familiares perciben que es más importante que sus hijos asistan a un centro de rehabilitación, debido a que esto les ha permitido mejorar sus condiciones de salud y por ende su bienestar físico. 
Se concluye que los apoyos tanto familiares, pedagógico y sociales, son una parte importante con respecto al mejoramiento de la calidad de vida de personas con discapacidad o algún tipo de dificultad, dado que estos van a favorecer en gran medida el bienestar personal y funcionamiento individual de cada persona.

\section{REFERENCIAS BIBLIOGRÁFICAS}

1. Alí S de los M, Blanco RL. Discapacidad intelectual, evolución social del concepto. Rev Fac Odontol Univ Nac (Cordoba) [Internet]. 2015 Apr 3 [cited 2019 May 26];8(1):38-41. Available from: http://revistas.unne.edu.ar/index.php/rfo/article/view/1631

2. Ríos MIH. El Concepto de Discapacidad: De la Enfermedad al. Rev CES Derecho [Internet]. 2015;6(2):46-59. Available from: http://www.scielo.org.co/pdf/cesd/v6n2/v6n2a04.pdf

3. Salud M de. ABECÉ de la discapacidad [Internet]. 2019. p. 1-27. Available from: https://www. minsalud.gov.co/sites/rid/Lists/BibliotecaDigital/RIDE/DE/PS/abece-de-la-discapacidad.pdf

4. Organización Mundial de la Salud OP de S. Clasificación Internacional del Funcionamiento, de la Discapacidad y de la Salud (CIF). 2001. 1-320 p.

5. Helena L, Agudelo L, Seijas V. La discapacidad en Colombia : una mirada global Colombia disability : a global perspective. Rev Col Med Fis Rehab [Internet]. 2012;22(2):164-79. Available from: http:// revistacmfr.org/index.php/rcmfr/article/view/64/61

6. Atención Integral de la Primera Infancia. Discapacidad en la primera infancia: una realidad incierta en Colombia. cero a siempre. 2013;5(5):32.

7. Clasificación de Tipo de Discapacidad - Histórica.

8. Carlos Egea García ASS. Clasificaciones de la OMS sobre discapacidad [Internet]. Artículos y Notas. 2001. p. 15-30. Available from: http://sid.usal.es/idocs/F8/ART6594/clasificacion_oms.pdf

9. Israel Cruz Velandia JHJ. Magnitud de la discapacidad en Colombia : una aproximación a sus determinantes. Artículo Orig [Internet]. 2008;6(3):23-35. Available from: https://revistas.urosario.edu. co/index.php/revsalud/article/view/490/431

10. Cesar J, Beltrán G, B CIG De. Discapacidad en Colombia : Reto para la Inclusión en Capital Humano. Discapac en Colomb Reto para la Inclusión en Cap Hum [Internet]. 2008;1:1-120. Available from: https://saldarriagaconcha.org/desarrollo_fsc/images/fsc/pdf/biblioteca_virtual/discapacidad/estadisticas_e_investigaciones/05_tomo_1_exclusion_DEPTAL_CH.pdf

11. Rey U. Universidad de concepción Campus Los Ángeles Escuela de Educación. :1-126.

12. Universidad de Santo Tomás (Bogota冈 CF de P, Téllez Castillo CA, Esguerra GA. Diversitas: perspectivas en psicología. [Internet]. Vol. 7, Diversitas: Perspectivas en Psicología. Bogotá, Colombia: Universidad Santo Tomás, Facultad de Psicología; 2011 [cited 2019 May 27]. 103-124 p. Available from: http://www.scielo.org.co/scielo.php?script=sci_arttext\&pid=S1794-99982011000100008

13. Fundación Miguel Unamuno y Jugo. CS-NMV-BK. Revista orbis : revista científica. [Internet]. Vol. 12, Orbis. Revista Científica Ciencias Humanas. Fundación Miguel Unamuno y Jugo; 2017 [cited 2019 May 25]. Available from: https://www.redalyc.org/html/709/70950101004/

14. Andrea P, Arbeláez R. Calidad de vida y educación : mirada contextual de niños, niñas y adolescentes bogotanos * Life and education : Context view. 2014;137-58.

15. Muñoz Cantero JM, Losada Puente L, Rebollo Quintela N. Calidad de vida y autodeterminación en alumnado con discapacidad incluido en aulas ordinarias. Rev Estud e Investig en Psicol y Educ. 2015;2(2):18.

16. Vazquez A, Cáceres N. El abordaje de la discapacidad desde la atención primaria de la salud. Ops. 2008. 162-171 p.

17. OMS; BANCO MUNDIAL. Informe Mundial sobre la Discapacidad 2011. Bibl la OMS. 2015;1349. 
18. Bortesi Longhi L. Calidad De Vida. Quipukamayoc. 2017;18(35):33.

19. Castro L, Antonio Casas J, Sánchez S, Vallejos V, Zúñiga D. Percepción de la calidad de vida en personas con discapacidad y su relación con la educación. Estud pedagógicos [Internet]. 2016 [cited 2019 May 25];42(2):39-49. Available from: http://www.scielo.cl/scielo.php?script=sci_arttext\&pid=S0718-07052016000200003\&lng=en\&nrm=iso\&tlng=en

20. Con P, Intelectual D. SC_Concepto calidad de vida por shalork y verdugo. :1-18.

21. Gil Llario MD, Morell Mengual V, Díaz Rodríguez I, Giménez García C, Ruiz Palomino E. Calidad de vida de los discapacitados intelectuales valorada por ellos mismos y los profesionales: variables implicadas. Int J Dev Educ Psychol Rev INFAD Psicol [Internet]. 2016 Jul 2 [cited 2019 May 26];1 (1):265. Available from: http://www.infad.eu/RevistaINFAD/OJS/index.php/IJODAEP/article/view/213

22. Vida CDE, Con DEP, Un D. Orione. 1(2012):19-46.

23. Social M de S y P. Sala situacional de las Personas con Discapacidad ( PCD ) Ministerio de Salud y Protección Social Oficina de Promoción Social [Internet]. 2018. p. 1-37. Available from: https:// www.minsalud.gov.co/sites/rid/Lists/BibliotecaDigital/RIDE/DE/PS/sala-situacional-discapacidad-junio-2018.pdf

24. Sotelo N, Lidia S, Domíguez S, Barboza M. Estudio comparativo de la calidad de vida de niños y adolescentes escolarizados de Lima y Ancash. Av en Psicol Rev la Fac Psicol y Humanidades [Internet]. 2013;21(1):65-71. Available from: http://www.unife.edu.pe/publicaciones/revistas/psicologia/2013/5 sotelo.pdf

25. Fernández-López JA, Fernández-Fidalgo M, Cieza A. Los conceptos de calidad de vida, salud y bienestar analizados desde la perspectiva de la clasificación internacional del funcionamiento (CIF). Rev Esp Salud Publica. 2010;84(2):169-84.

26. Centro Español de Documentación sobre Discapacidad L, Gómez Sánchez LE, Alcedo Rodríguez MÁ. Revista española de discapacidad. [Internet]. Vol. 3, Revista Española de Discapacidad (REDIS), ISSN-e 2340-5104, Vol. 3, No. 1, 2015 (Ejemplar dedicado a: REVISTA ESPAÑOLA DE DISCAPACIDAD), págs. 77-91. Centro Español de Documentación sobre Discapacidad (CEDD) del Real Patronato sobre Discapacidad y FundaciónEguía Careaga; 2015 [cited 2019 May 26]. 77-91 p. Available from: https:// dialnet.unirioja.es/servlet/articulo?codigo $=5151750$

27. Urzúa M. A, Cortés R. E, Prieto C. L, Vega B. S, Tapia N. K. Autoreporte de la calidad de vida en niños y adolescentes escolarizados TT - Self-report of quality of life in school age children and adolescents. Rev Chil Pediatr [Internet]. 2009;80(3):238-44. Available from: http://www.scielo.cl/scielo. php?script $=$ sci_arttext\&pid=S0370-41062009000300005

28. Steinhardt LC, Onikpo F, Kouamé J, Piercefield E, Lama M, Deming MS, et al. Integrated management of childhood illness ( IMCI) strategy for children under five (Review) SUMMARY OF FINDINGS FOR THE MAIN COMPARISON. PLoS One [Internet]. 2015;9(1):276. Available from: http://www.ncbi.nlm. nih.gov/pubmed/15608935\%0Ahttp://www.biomedcentral.com/1471-2458/14/277\%0Ahttp://www. ijhpm.com/article_2944_616.html\%0Ahttp://www.pubmedcentral.nih.gov/articlerender.fcgi?artid=3563136\&tool=pmcentrez\&rendertype=abstract\%0Ahttp://www.sc

29. Información estadística de la discapacidad [Internet]. Información Estadística de la Discapacidad. 2004. p. 1-41. Available from: https://www.dane.gov.co/files/investigaciones/discapacidad/inform_estad.pdf

30. Lucas Correa Montoya MCCM. Discapacidad e Inclusión Social en Colombia. Informe alternativo de la Fundación Saldarriaga Concha al Comité de las Naciones Unidad sobre los derechos de las personas con discapacidad. In: Editorial Fundación Saldarriaga Concha [Internet]. 2016. p. 1-162. Available from: https://www.saldarriagaconcha.org/wp-content/uploads/2019/01/pcd_discapacidad_inclusion_social.pdf

31. Colombia EC de. LEY ESTATUTARIA 1618 DE 2013 (febrero 27). 2013;2013(febrero 27). Available from: https://discapacidadcolombia.com/phocadownloadpap/LEGISLACION/LEY ESTATUTARIA 1618 DE 2013.pdf

32. Martinez E. Test para dos muestras independientes Test de Mann-Whitney-Wilcoxon. Métodos No Paramétricos I. 2013;2:81-97. 
33. Salud MDE, Salud ELMDE. Resolución 8430 de 1993. República Colomb [Internet]. 1993;(Octubre 4):1-19. Available from: https://www.minsalud.gov.co/sites/rid/Lists/BibliotecaDigital/RIDE/DE/ DIJ/RESOLUCION-8430-DE-1993.PDF

34. Patricia C, Lema H. CALIDAD DE VIDA Y SITUACIÓN DE DISCAPACIDAD. Rev hacia la promoción la salud. 2009;14:112-25.

35. Córdoba Andrade L, Henao Lema CP, Verdugo Alonso MÁ. Calidad De Vida De Adultos Colombianos Con Discapacidad Intelectual. Hacia la promoción la salud [Internet]. 2016;0121-7577(24628425):91-105. Available from: http://200.21.104.25/promocionsalud/downloads/Revista21(1)_8.pdf

36. GONZÁLEZ MARTÍN E, GÓMEZ SÁNCHEZ LE, ALCEDO RODRÍGUEZ M. Á. Enfermedades raras y discapacidad intelectual: evaluación de la calidad de vida de niños y jóvenes. Siglo Cero Rev Española sobre Discapac Intelect. 2016;47(3):7.

37. Exteriores MDR, Nacional MDD, Trabajo M De, Comercio M De, Vivienda M De, Transporte M De, et al. Conpes Social [Internet]. Consejo Nacional de Política Económica y Social. 2013. Available from: https://colaboracion.dnp.gov.co/CDT/Conpes/Social/166.pdf

38. URZÚA M A, CAQUEO-URÍZAR A, ALBORNOZ B N, JARA S C. Calidad de vida en la infancia: estudio comparativo entre una zona rural y urbana en el norte de Chile. Rev Chil pediatría [Internet]. 2013 Jun [cited 2019 May 25];84(3):276-84. Available from: http://www.scielo.cl/scielo.php?script=sci_arttext\&pid=S0370-41062013000300005\&lng=en\&nrm=iso\&tlng=en

39. Fernandez-Gabalón C. La Discapacidad Intelectual en la sociedad. 2013;152. Available from: http://diposit.ub.edu/dspace/bitstream/2445/53928/1/Clara_Fernandez_Gabalon.pdf

40. MINSALUD M de S y PS. Ley estatutaria 1618 del 2013. Derechos de las Personas con Discapacidad. 2013;41. Available from: https://www.minsalud.gov.co/sites/rid/Lists/BibliotecaDigital/RIDE/DE/ PS/documento-balance-1618-2013-240517.pdf

41. Gallego CAM, Parada YPJ, Nieto APA, Brito YR, Redondo AL, Ascanio J, et al. Determinantes Sociales En Salud: Construccion De Conocimiento A Traves De La Cartografia Social. Rev Científica Signos FónicoS [Internet]. 2015 Oct 1;1(3).

42. Gallego CAM, Redondo AL, Cabeza JAM, Gil DMD, Carvajal SMY, Clavijo ZA, et al. Discapacidad: Una Perspectiva Desde Los Ejes De Desigualdad. Rev Científica Signos Fónicos [Internet]. 2017 Oct 25;2(3).

43. Redondo AL, Gallego CAM, Florez LLP, Machuca YGC, Mejia KLH, Jerez LVM, et al. Relacion Entre La Discapacidad Y La Desigualdad De La Clase Social. Rev Científica Signos Fónicos [Internet]. 2017 Oct $25 ; 2(3)$. 